\title{
Tratamento Cirúrgico da Ruptura do Manguito Rotador Através da Transferência do Grande Dorsal Artroscopicamente Assistida
}

\author{
Surgical Treatment of Rotator Cuff Rupture Through Arthroscopically Assisted Latissimus \\ Dorsi Transfer
}
Tratamiento Quirúrgico de la Ruptura del Manguito Rotador a través de la Transferencia del Gran Dorsal Artroscópicamente Asistida

\begin{abstract}
Cristiano Benicio dos Santos ${ }^{1 *}$, Kemilson Aparecido Brito de Sousa ${ }^{1}$, Rafael Patrocínio de Paula Costa ${ }^{1,2}$, Maria Luzete Costa Cavalcante ${ }^{1,2}$, Ana Vitória Martins Pimentel ${ }^{3}$, Ângelo Gonçalves de Araújo ${ }^{3}$, Marcio de Paiva Bezerra Carrah ${ }^{1}$
\end{abstract}

\section{RESUMO}

Introdução: As lesões do manguito rotador correspondem até 70\% das queixas álgicas no ombro, sendo um meio significativo de disfunção do membro. Existem diferentes formas de classificação para as lesões do manguito rotador, entre elas a classificação de Cofield que avalia conforme a extensão da lesão como: pequena, moderada, grande e maciça. Ademais, algumas lesões maciças podem ser classificadas como irreparáveis. Assim, a transferência do latíssimo do dorso por via artroscópica é uma opção cirúrgica para os pacientes com lesão irreparável, pois proporciona o alívio da dor e recuperação de parte da funcionalidade, além de menor dano tecidual e menos complicações pós-operatórias. Detalhamento do caso: Este relato de caso disserta sobre um paciente de 61 anos, aposentado pela construção civil, que após atropelamento, iniciou quadro de dor intensa e limitação do arco de movimento do ombro direito, sendo diagnosticado com ruptura do manguito rotador. $\mathrm{O}$ tratamento usado foi a transferência do grande dorsal assistida por artroscopia. Foi realizado uma avaliação funcional pré e pósoperatória através das escalas UCLA e ASES, obtendo com isso um prognóstico bom do quadro clínico do paciente. Discussão: Dentro do trabalho, foram destacadas as indicações para a escolha do procedimento e avaliado 0 desfecho clínico quando comparado com a literatura atual.

Palavras-Chave: Lesões do Manguito rotador, Manguito rotador/cirurgia, Artroscopia.

\begin{abstract}
Introduction: Rotator cuff injuries account for up to $70 \%$ of painful shoulder complaints and can cause limb dysfunction. There are different forms of classification for the rotator cuff lesions, among them, the Cofield classification that evaluates according to the extent of the lesion as: small, moderate, large and massive. In addition, some massive lesions can be classified as irreparable. Thus, the transfer of the latissimus dorsi through the arthroscopic route is a surgical option for patients with irreparable damage, as it provides relief of pain and recovery of part of the functionality, in addition to less tissue damage and fewer postoperative complications. Case report: This case report discuss a 61-year-old patient, retired by the civil construction, who, after being run over, initiated severe pain and limiting the range of motion of the right shoulder, being diagnosed with rupture of the rotator cuff. The treatment used was the transfer of the latissimus dorsi assisted by arthroscopy. A preoperative and postoperative functional evaluation was performed through the UCLA and ASES scales, thus obtaining a good prognosis of the clinical picture of the patient. Discussion: During the research, the indications for choosing the procedure were highlighted and the clinical outcome was evaluated when compared to the current literature.
\end{abstract}

Key words: Rotator Cuff Injuries, Rotator Cuff/surgery, Arthroscopy.

\footnotetext{
1 Programa de Residência Médica em Ortopedia e Traumatologia pela Universidade Federal do Ceará (UFC), Fortaleza-CE. *E-mal: crisbenicio1016@gmail.com

${ }^{2}$ Hospital Universitário Walter Cantídio da Universidade Federal do Ceará (UFC), Fortaleza-CE.

${ }^{3}$ Universidade de Fortaleza (UNIFOR), Fortaleza-CE.
} 


\section{RESUMEN}

Introducción: Las lesiones del manguito rotador corresponden hasta un $70 \%$ de las quejas álgicas en el ombro, siendo un medio significativo de disfunción del miembro. Existen diferentes formas de clasificación para las lesiones del manguito rotador, entre ellas la clasificación de Cofield que analiza conforme a la extensión de la lesión como: pequena, moderada, grande e maciza. Ademas, algunas lesiones macizas pueden ser clasificadas como irreparables. Asi, la transferencia del latísimo del dorso por via artroscópica es una opción quirúrgica para los pacientes con lesión irreparable, pues proporciona un alivio del dolor y recuperación de parte da la funcionalidad, ademas de menor daño tecidual y menos complicaciones pós-operatórias. Detallado del caso: Este relato de caso diserta sobre un paciente de 61 años, jubilado por la construcción civil, que después de un atropellamiento, inicio un quadro de dolor intenso y limitación del arco de movimento del ombro derecho, siendo diagnosticado con ruptura del manguito rotador. El tratamiento utilizado fue la transferencia del grande dorsal asistido por artroscopia. Fue realizado una evaluación funcional pré y pós-operatório através de las escalas UCLA y ASES, obteniendo así un prognóstico bueno del cuadro clínico del paciente. Discusión: Dentro del trabajo, fueron destacados las indicaciones para a elección del procedimento y evaluado el resultado clínico cuando comparado con la literatura actual.

Palabras clave: Lesiones del Manguito de los Rotadores, Manguito de los Rotadores/cirugía, Artroscopía.

\section{INTRODUÇÃO}

O manguito rotador é constituído pelos músculos: supra-espinhal, infra-espinhal, redondo menor e subescapular. Essa estrutura é responsável pela estabilização dinâmica do ombro, mantendo-o centralizado na cavidade glenoidal. Além disso, realiza os principais movimentos do ombro como: elevação, rotação externa, rotação interna e abdução. (METZKER A, 2010).

Na prática ortopédica, as lesões do manguito rotador correspondem até $70 \%$ das queixas álgicas no ombro (LITCHINA A, et al., 2015), além de representar um meio significativo de disfunção do membro. As lesões acometem, principalmente, pacientes entre 40 a 60 anos e podem ocorrer por causas traumáticas ou degenerativas (VIEIRA F, et al., 2015). Existem diferentes formas de classificação para as lesões do manguito rotador, entre elas, a classificação de Cofield. Esta se divide de acordo com a extensão da lesão, descrita como pequena $(<1 \mathrm{~cm})$, moderada (entre 1 e $3 \mathrm{~cm}$ ), grande (entre 3 e $5 \mathrm{~cm}$ ) e maciça $(>5 \mathrm{~cm})$. (LITCHINA A, et al., 2015; ANDRADE R, et al., 2004).

As lesões maciças podem ser classificadas como lesões irreparáveis quando acometem 2 ou mais tendões e são associadas a retração do tendão ao nível da glenóide, classificadas como Patte 3 . Além disso, músculos com infiltração gordurosa acentuada (Goutallier 3 ou 4) e atrofia muscular importante, estágio 3 de Thomazeau 5 (STRUHL S., 2002), não apresentam bom prognóstico para a redução anatômica devido à alta incidência de re-ruptura. (ANDRADE R, et al., 2004; ELHASSAN, et al., 2016).

No reparo cirúrgico das lesões irreparáveis, a restauração da continuidade tecidual é difícil e apresenta resultados insatisfatórios com uso isolado de âncoras. No entanto, a reconstrução do manguito, no caso das lesões irreparáveis, pode aliviar a dor, mas com perda funcional do ombro. (WARNER J e PARSONS I, 1999).

Desse modo, a transferência do latíssimo do dorso é uma técnica introduzida no arsenal terapêutico em 1988, por Gerber et al. Para tratamento de pacientes com lesão irreparável do manguito. Após a transferência, o latíssimo do dorso passa a funcionar como um depressor da cabeça e um rotador externo ativo. Assim, a transferência do grande dorsal para lesões póstero-superiores se apresenta como uma opção viável, proporcionando alívio da dor e recuperação de parte da funcionalidade. (ANDRADE R, et al., 2004; STRUHL S, 2002).

Por fim, esse trabalho visa apresentar o caso de um paciente, 61 anos, sexo masculino, com ruptura de manguito rotador de origem traumática, apresentando grande retração e infiltração gordurosa. 


\section{ESTUDO DE CASO}

O paciente, 61 anos, aposentado pela construção civil, refere que após atropelamento, iniciou quadro de dor intensa e limitação do arco de movimento (ADM) do ombro direito, tendo procurado assistência médica, não sendo identificado fraturas resultantes do trauma.

Todavia, após um ano do acidente, deu entrada no serviço de ortopedia especializado em cirurgia do ombro apresentando persistência dos sintomas citados anteriormente. No exame físico, apresentou atrofia na região correspondente à musculatura do supra-espinhal e infra-espinhal, quando comparada ao lado contralateral. Além disso, mostrou uma diminuição importante da ADM do ombro direito, conforme tabela 1 abaixo:

Tabela 1 - Avaliação Pré-operatória do arco de movimento.

\begin{tabular}{llll}
\hline & Ombro direito & Ombro esquerdo \\
\hline Elevação ativa & 70 graus & 170 graus \\
\hline $\begin{array}{l}\text { Rotação externa com } \\
\text { junto ao corpo }\end{array}$ & o cotovelo & 40 graus & 80 graus \\
\hline Rotação interna ativa & L1 & T5 \\
\hline
\end{tabular}

Fonte: Próprio autor.

O paciente apresentou positividade aos seguintes testes especiais: sinal da cancela, de Jobe, de Gerber e do corneteiro. Ademais, obteve 43 pontos na escala de ASES e 18 pontos na escala de UCLA, caracterizando função ruim do ombro direito.

Apresentou achados radiográficos compatíveis com ruptura extensa do manguito rotador, com ascensão da cabeça umeral e com espaço subacromial menor que $7 \mathrm{~mm}$, associado à perda do arco gótico do ombro (Figura 1).

Figura 1 - Avaliação radiográfica Pré-operatória.
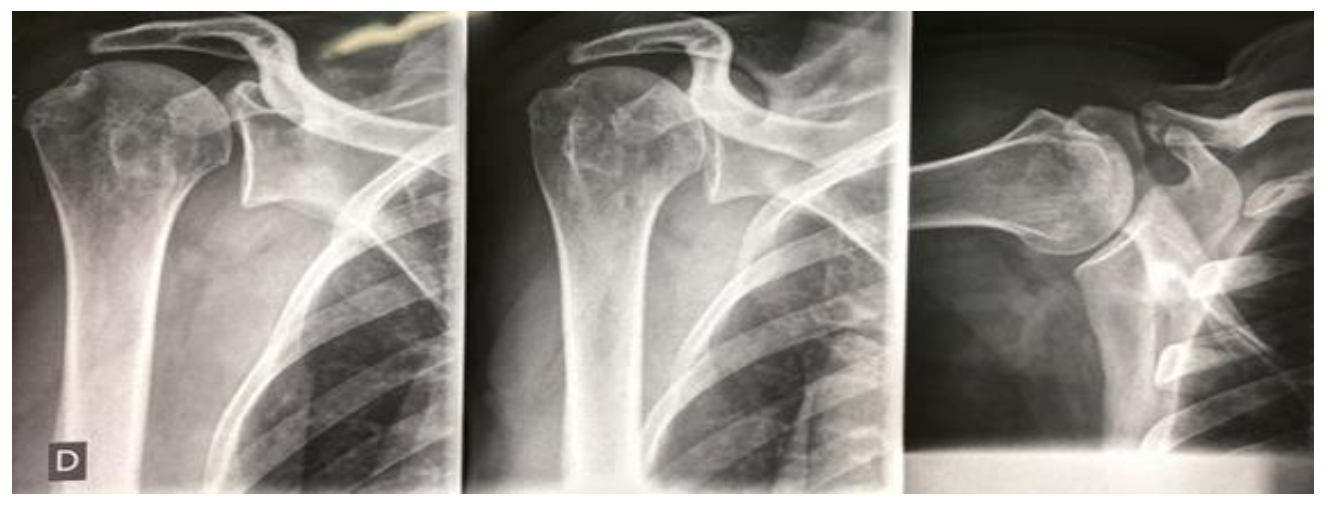

Fonte: Próprio autor.

Na ressonância magnética do ombro (Figura 2), encontramos uma ruptura completa do tendão supraespinhal com retração importante até nível da articulação acrômio-clavicular, sendo classificada como Patte 3. Foi encontrada também ruptura parcial do infra-espinhal, artrose acrômio-clavicular e bursite subacromialsubdeltóide. 
Figura 2 - Imagem de ressonância magnética obtida no pré-operatório.

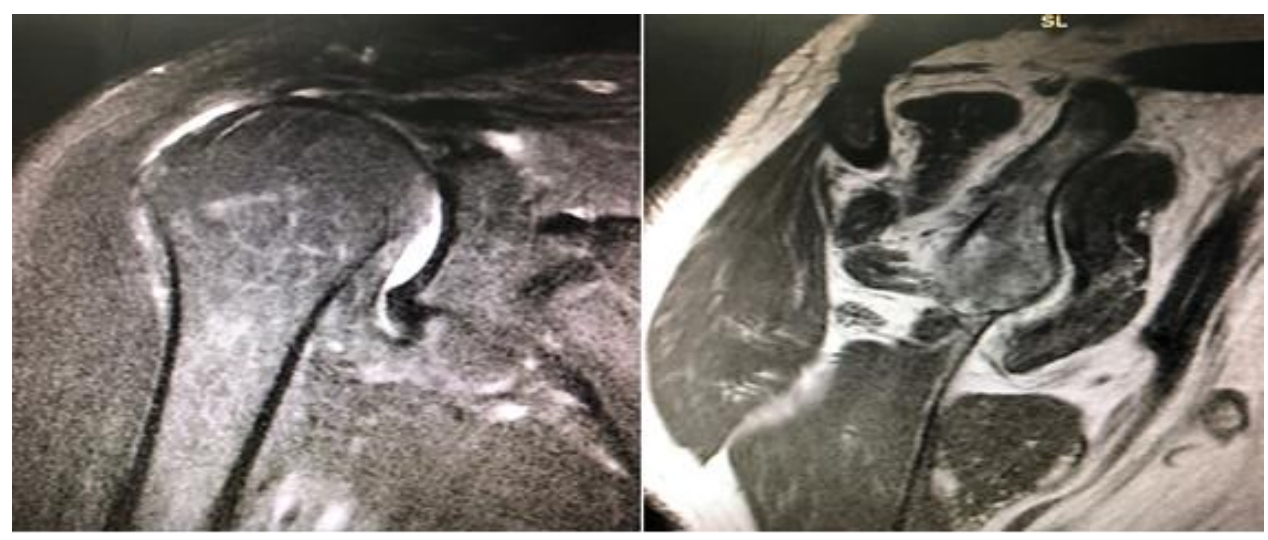

Fonte: Próprio autor.

Desta forma, foi indicado cirurgia de transferência muscular do grande dorsal para região de "footprint" da cabeça umeral, artroscopicamente assistida.

Na cirurgia, o paciente foi submetido à anestesia geral e bloqueio de plexo braquial, realizou-se então a assepsia, antissepsia e posicionamento de campos estéreis. O paciente foi colocado em posição lateral com o membro a ser abordado em tração.

Inicialmente, foi realizado acesso posterior axilar com dissecção do grande dorsal, sendo realizada a tenotomia do mesmo. Depois, em segundo tempo operatório, realizou-se a confecção dos portais artroscópicos, sendo identificada lesão extensa do supra-espinhal e infra-espinhal, de $5,0 \times 2,0 \mathrm{~cm}$. Além disso, o fechamento completo e sem tensão da lesão foi feito com suturas usando três âncoras 5.0 e utilizando dois pontos do tipo "Mason-Allen" e dois pontos do tipo "Mattress".

Foi realizada tenotomia do bíceps associado à sua tenodese, seguida da passagem do tendão do grande dorsal para a região da tuberosidade maior, sendo realizada sua fixação através de âncora 5.0.

$\mathrm{Na}$ avaliação intra-operatória foi evidenciado o fechamento do manguito sem tensão e realizada sutura por planos com aplicação de curativo estéril local, e o uso da tipóia de abdução com imobilização do membro superior direito.

No período de seguimento ambulatorial, o paciente evoluiu sem complicações imediatas, tal como infecções locais ou deiscências. Ademais, apresentou boa cicatrização local e foi mantido em tipóia de abdução por período de quarenta e cinco dias, seguido por reabilitação fisioterápica.

Vale destacar que em seu retorno após 6 meses da cirurgia, não apresentou queixas álgicas (EVA 1/10) e obteve melhora do ADM, como mostra a tabela 2 abaixo:

Tabela 2 - Avaliação do arco de movimento pós-operatório.

\begin{tabular}{lll}
\hline & Ombro direito & Ombro esquerdo \\
\hline Elevação ativa & 180 graus & 180 graus \\
\hline $\begin{array}{l}\text { Rotação externa ativa com cotovelo } \\
\text { junto ao corpo }\end{array}$ & 60 graus & 90 graus \\
\hline Rotação interna ativa & T8 & T8 \\
\hline
\end{tabular}

Fonte: Próprio autor. 
No pós-operatório, foi solicitado novo estudo radiográfico (Figura 3), no qual pode-se observar um nítido aumento do espaço subacromial.

No seguimento ambulatorial, após seis meses de pós-operatório, apresentou melhora significativa da funcionalidade do ombro, o que foi conferida pelas escalas de ASES e UCLA, na qual obteve a de 90 e 35, respectivamente, demonstrando o sucesso do procedimento cirúrgico.

\section{DISCUSSÃO}

No caso descrito, observamos uma ruptura traumática crônica, com grande retração do tendão, associada à infiltração gordurosa avançada, sugerindo um resultado ruim para o reparo isolado do tendão.

No pré-operatório, deve-se considerar aspectos anatomopatológicos combinados com a extensão da lesão e sua topografia nos planos sagital e frontal, além da qualidade trófica do músculo e funcionalidade do ombro, visto que tais fatores influenciam na indicação e prognóstico cirúrgico (PATTE, 1989).

A ruptura do manguito rotador do paciente foi classificada como Patte III devido à retração do supra-espinhal no plano frontal. No plano sagital, a ruptura possuía mais de $5 \mathrm{~cm}$, acarretando a ausência quase total do manguito. Neste grau, a cabeça do úmero migra cranial e frontalmente, devido à ausência de oposição do músculo deltoide, colidindo contra o arco coracoacromial, exigindo tratamento cirúrgico precoce (PATTE, 1989).

Umas das escalas utilizadas na avaliação da função do ombro do paciente com lesão do manguito rotador é da University of California at Los Angeles (UCLA). Ela é terceira mais usada nos estudos publicados sobre manguito rotador nas revistas ortopédicas. Apresenta confiabilidade moderada a muito alta (MALAVOLTA E, et al., 2017). A avaliação da dor (10 pontos), função (10 pontos), amplitude da flexão anterior ativa (5 pontos), teste de força manual para flexão anterior (5 pontos) e satisfação do paciente (5 pontos), a soma de todos esses itens equivale a 35 pontos. Os resultados conforme a pontuação da escala são: excelente (34-35), bom (28-33), razoável (21-27) e ruim (20-0). (OKU, et al., 2006). O paciente apresentava no pré-operatório 18 pontos no escore UCLA e no pós-operatório 35 pontos.

O paciente também foi avaliado pela escala ASES, que avalia a funcionalidade do ombro, esta escala varia de 0 a 100 pontos. Desse modo, uma pontuação menor que 20 pontos é considerado como péssimo, 21 a 40 pontos é ruim, 41 a 60 pontos é regular, 61 a 80 pontos é bom e 81 a 100 pontos é excelente (FRANÇA FO, et al., 2018). O paciente no pré-operatório tinha 43 pontos e no pós-operatório 90 pontos, demonstrando a melhora funcional após a cirurgia.

Vale ressaltar que a infiltração gordurosa é um fator importante para a indicação cirúrgica, pois quando a infiltração gordurosa é estágio 2,3 ou 4 de Goutallier a taxa de falha no reparo cirúrgico após a cirurgia é maior que $50 \%$. Todavia, se a infiltração for estágio 0 ou 1 a taxa de falha é em torno de $10 \%$ (GOUTALLIER D, et al., 1994). O paciente apresentava degeneração gordurosa estágio 3 de Goutallier, logo é um paciente que apresenta chances consideráveis de falha no reparo cirúrgico.

Embora a técnica artroscópica apresente pontos negativos como a necessidade de uma curva de aprendizado maior, essa técnica permite uma visualização adequada associada a um dano tecidual mínimo, o que acarreta em menor sangramento e menor morbidade, apresentando menor número de complicações quando comparado com a técnica aberta 1011 1. (VEADO MADC, et al., 2011; MIYAZAKI AN, et al., 2009; VEADO MADC, et al., 2008) 
Os resultados obtidos com a transferência do latíssimo do dorso possuem uma boa durabilidade. Em estudo realizado por El-Azab et al. (2015), identificou-se que $86 \%$ dos pacientes que foram submetidos ao reparo cirúrgico com transferência de latíssimo do dorso referiram bons resultados após cerca de 10 anos do procedimento. (EL-AZAB, et al., 2015; HART, et al., 2010)

\section{CONSIDERAÇÕES FINAIS}

Desse modo, percebe-se que a transferência do latíssimo do dorso artroscopicamente assistida apresenta um excelente resultado funcional e menores complicações no pós-operatório. Logo, é uma opção adequada para o tratamento de lesões maciças e irreparáveis do manguito rotador.

\section{REFERÊNCIAS}

1. ANDRADE RP, et al. Lesões do manguito rotador. Rev. bras. ortop, v. 39, n. 11/12, p. 621-636, 2004.

2. CARVALHO AL, et al. Rotator cuff injuries and factors associated with reoperation. Revista Brasileira de Ortopedia (English Edition), v. 51, n. 3, p. 298-302, 2016.

3. VEADO MADC, et al. Prospective and comparative study on functional outcomes after open and arthroscopic repair of rotator cuff tears. Revista Brasileira de Ortopedia (English Edition), v. 46, n. 5, p. 546-552, 2011.

4. VEADO MADC, et al, et al. Functional evaluation of arthroscopic repairs of complete rotator cuff tears associated to acromioplasty. Revista Brasileira de Ortopedia, v. 43, n. 11-12, p. 505-512, 2008.

5. FRANÇA FDO, et al. Avaliação clínica e funcional de pacientes submetidos a artroplastia reversa com seguimento mínimo de um ano. Revista Brasileira de Ortopedia, 2018

6. ELHASSAN BT, et al. Outcome of lower trapezius transfer to reconstruct massive irreparable posterior-superior rotator cuff tear. Journal of shoulder and elbow surgery, v. 25, n. 8, p. 1346-1353, 2016.

7. EL-AZAB HM, et al. Long-term follow-up after latissimus dorsi transfer for irreparable posterosuperior rotator cuff tears. JBJS, v. 97, n. 6, p. 462-469, 2015.

8. GOUTALLIER D, et al. Fatty muscle degeneration in cuff ruptures. Pre-and postoperative evaluation by CT scan. Clinical orthopaedics and related research, n. 304, p. 78-83, 1994.

9. HART R, et al. Latissimus dorsi transfer for the treatment of irreparable craniodorsal tears of the rotator cuff. Acta chirurgiae orthopaedicae et traumatologiae Cechoslovaca, v. 77, n. 3, p. 215-221, 2010.

10. MALAVOLTA EA, et al. Correlação entre as escalas da UCLA e Constant-Murley nas roturas do manguito rotador e fraturas da extremidade proximal do úmero. Revista Brasileira de Ortopedia, v. 53, n. 4, p. 441-447, 2018. ok

11. METZKER CAB. Tratamento conservador na síndrome do impacto no ombro. Fisioterapia em movimento, v. 23, n. 1, 2010.

12. MIYAZAKI NA, et al. Lesões extensas do manguito rotador: avaliação dos resultados do reparo artroscópico. Rev Bras Ortop, v. 44, n. 2, p. 148-52, 2009.

13. OKU EC, et al. Tradução e adaptação cultural do Modified-University of California at Los Angeles Shoulder Rating Scale para a língua portuguesa. Revista Brasileira de Reumatologia, 2006.

14. PATTE D. Classification of rotator cuff lesions. Clinical orthopaedics and related research, n. 254, p. 81-86, 1990.

15. STRUHL S. Anterior internal impingement: an arthroscopic observation. Arthroscopy-the Journal of Arthroscopic and Related Surgery, v. 18, n. 1, p. 2-7, 2002.

16. VIEIRA, FA, et al. Lesão do manguito rotador: tratamento e reabilitação. Perspectivas e tendências atuais. Revista Brasileira de Ortopedia, v. 50, n. 6, p. 647-651, 2015.

17. WARNER JJ, PARSONS IMIV. Latissimus dorsi tendon transfer: a comparative analysis of primary and salvage reconstruction of massive, irreparable rotator cuff tears. Journal of shoulder and elbow surgery, v. 10, n. 6, p. 514-521, 2001. 